\title{
Adaptation of Web Pages and Images for Mobile Applications
}

\author{
Stephan Kopf, Benjamin Guthier, Hendrik Lemelson, Wolfgang Effelsberg \\ Department of Computer Science IV, University of Mannheim, Mannheim, Germany
}

\begin{abstract}
In this paper, we introduce our new visualization service which presents web pages and images on arbitrary devices with differing display resolutions. We analyze the layout of a web page and simplify its structure and formatting rules. The small screen of a mobile device is used much better this way. Our new image adaptation service combines several techniques. In a first step, border regions which do not contain relevant semantic content are identified. Cropping is used to remove these regions. Attention objects are identified in a second step. We use face detection, text detection and contrast based saliency maps to identify these objects and combine them into a region of interest. Optionally, the seam carving technique can be used to remove inner parts of an image. Additionally, we have developed a software tool to validate, add, delete, or modify all automatically extracted data. This tool also simulates different mobile devices, so that the user gets a feeling of how an adapted web page will look like. We have performed user studies to evaluate our web and image adaptation approach. Questions regarding software ergonomics, quality of the adapted content, and perceived benefit of the adaptation were asked.
\end{abstract}

Keywords: Content repurposing, image adaptation, adaptation of web pages, mobile applications

\section{INTRODUCTION}

Although a great number of people in developed countries use mobile devices, the number of applications which are available on all the different mobile device classes is still relatively low. Usually, applications are only developed and tested for a certain device class (e.g., personal digital assistant, PDA) and have to be adapted for other device classes (e.g., mobile phones). The porting of applications to the wide variety of mobile device classes is a time consuming issue. Additionally, testing and maintainability of applications is getting cumbersome for such a huge number of different mobile device classes.

A major challenge for the development of mobile applications is the heterogeneity of the different device classes. In the Mobile Business project, ${ }^{1}$ we have built a generic software platform that supports the development of context-sensitive services for service brokers and providers. The client framework facilitates the implementation of generic client applications for mobile devices that enable a dynamic integration and execution of new services. To illustrate and evaluate the functionality of our framework we have built two sample applications - a restaurant finder and a bargain hunter. These applications are context-sensitive, because they intend to deliver services or information to the user that best fit the current context. For instance, in case of the restaurant finder application, it would be helpful if the user's current position, his preferences like 'non-smoking', 'prefer Italian food', 'outdoor seats', or 'not too expensive' are considered. If the calendar of a user indicates other appointments in the near future the proposed restaurants should not be too far away (depending on available transportation). In most cases, our sample applications use web pages for presentation. It would not be possible to visualize the content in a suitable way without the support of automatic adaptation techniques.

To enable the fast and efficient implementation of new applications for mobile devices, we have implemented and integrated several services in our framework, e. g., a service discovery technique to automatically identify relevant services based on user preferences and user context; a Wireless-LAN-based indoor positioning service which reliably estimates the position of a mobile user inside a building, and a visualization service to adapt multimedia content to different mobile devices and present it in a suitable way. In this paper, we introduce our new visualization service to present web pages and images on mobile devices with differing display resolutions. The adaptation service must guarantee that even in case of very small displays the relevant content can be recognized.

A very important characteristic of mobile devices is the limited resolution of the display. If images or web pages are presented on mobile devices in a naïve way, the content cannot be recognized very well on these small displays, and the 
interaction with the application would be inefficient because the user has to scroll the web page horizontally and vertically. A good adaptation service would select the relevant parts of an image or a web page to be visualized.

In the following, we will present our new algorithms for the adaptation of images and web pages. The outline of the paper is as follows: The following Section gives an overview of previous work in the context of adaptation systems for images and web pages. Major challenges are presented in Section 3. Sections 4 and 5 illustrate our new adaptation algorithms. In Section 6, we present experimental results and user evaluations, and conclude the paper in Section 7.

\section{RELATED WORK}

Many ideas were published in the last years in the context of adaptation for small, mobile devices. Before we can build an adaptation system, we have to consider the environment the system the system will be used in and derive some basic properties:

- Location of the adaptation system: We can distinguish server-, proxy- or client-based systems. Server-based adaptation $^{2,3}$ may result in a performance bottleneck if a large number of clients request data at the same time and if the complexity of the adaptation algorithm is high. Proxies ${ }^{4,5}$ can reduce these disadvantages, but usually increase the overall complexity of the architecture. Client-based adaptation ${ }^{6,7}$ is unsuitable in many cases due to the limited memory, CPU power, capacities of wireless networks and the request for energy efficiency. We use a server-based system which is not only suitable for the adaptation of images and web pages, but supports audio streams and videos, too. 8,9

- Considered hardware of mobile devices: Most adaptation systems support the resolution of the display ${ }^{10,11}$ and indirectly consider the limited amount of memory, capacities of wireless networks and CPU power. ${ }^{12}$ Other features like the color depth of the display, ${ }^{13,14}$ available network capacities or energy consumption are relevant in some scenarios, too. We focus on the resolution of the target display. Other restrictions are of minor relevance for our system, because only few resources of the mobile device are used (server-based adaptation), and we do not have to handle a large amount of data, e.g., in contrast to video adaptation.

- Realtime adaptation: The adaptation system could adapt the content on the fly or use precalculated versions of the adapted data that is stored on a server. We assume that it is not possible to guess all expected target resolutions of the images a user might request in advance. In case of web pages, it is important that the content is up-to-date. Therefore, the content is adapted on the fly. Nevertheless, we can precalculate some data in advance, e.g. to store additional metadata which describes the semantic content of images.

- User preferences and user context: The complexity of an adaptation system might increase significantly if the context of the user is regarded. Privacy issues might arise because a user might not want to submit personal data over wireless network connections. In our system, the user can specify preferences which affect the adaptation algorithm. E.g., the importance of semantic features like faces can be specified manually.

- Semi-automatic adaptation: It is also very relevant to decide whether the adaptation system is automatic or semi-automatic (supports user interaction). Our system can adapt multimedia content automatically, but we have developed a software tool which makes the validation of the automatically extracted data much easier.

\subsection{Adaptation of web pages}

To archive a suitable presentation of the content of web pages, a minimum resolution and size of the display is recommended. Manual adaptation causes high costs and is not economical in most cases. Many approaches have been proposed to adapt web content to the limited screen resolution of mobile devices. ${ }^{15-20} \mathrm{~A}$ common technique is to convert an HTML file into an XML notation and derive an individual presentation for a mobile device. ${ }^{21,22}$ Huang and Sundaresan ${ }^{23}$ identify functional objects and transform them into an XML representation. The advantage of their approach is that the semantic concept of web pages can be captured and a new combination of the data based on transaction schemas is feasible. On the other hand, rules have to be defined to extract the functional objects for each web page.

Chen et al. ${ }^{24}$ have presented an approach which changes the structure of a web page and splits it into smaller units. An overview page with self-explanatory links supports the navigation to the subpages. Maekawa et al. ${ }^{25}$ extend this approach and use automatic scrolling for sub pages which are still too large to be presented on a mobile device. Users can set the 
preferred scrolling speed. Blekas et al. ${ }^{26}$ use RSS data and present it on a dynamically generated index page. This approach reduces the data size significantly, and a concise title with corresponding text is directly available in the RSS data. A major disadvantage in their approach is the fact that images are not considered.

\subsection{Adaptation of images}

The adaptation of images is also very important for the presentation of web pages on small displays. Simple image adaptation techniques like scaling or cropping do not provide satisfactory results in many cases. Especially for the adaptation of images in web pages, it might be useful to specify categories of images like icons, advertisements or essential content and use a suitable adaptation for each category. ${ }^{27}$

Several algorithms have been proposed to adapt the resolution of images. The overall goal is to present images in a way so that the content can be recognized easily. ${ }^{28,29}$ Horizontal and vertical scrolling of images is inefficient and should be avoided. On the other hand, scaling based techniques might reduce the size of the image and relevant content might no longer be recognizable. Most approaches identify attention objects which incur the interest of users. The relevance of an object is defined by its attention value. If several attention objects are adjacent in an image they are combined into a region of interest. Most approaches merely differ in how they present one or more regions of interest.

Suh et al. ${ }^{30}$ use cropping to generate thumbnail images based on a saliency map ${ }^{31}$ which describes the visual relevance of image regions for users. Santella et al. ${ }^{32}$ use eye tracking to identify relevant regions. Setlur et al..$^{33}$ identify regions, merge similar ones, and specify the relevance of each region. The adaptation is done by removing irrelevant regions at the image borders. If the image is still too large, they use a so-called inpainting technique. By using this technique, an empty image of the requested size is created and the relevant objects are arranged within the image. The remaining holes between the objects are filled by scaling unused regions of the original image.

Liu et al..$^{34}$ use automatic browsing to present large images on small displays. The images are visualized as automatic slide shows. The selection of relevant regions is based on saliency maps, as well as text and face recognition. The parameters minimal perceptive size and minimal perceptive time define the acceptable size for an attention object and the duration to present each image. Other approaches warp large images, e. g., the fisheye view warping from Liu and Gleicher. ${ }^{35}$ They assume that one region in the image is most important. This region is left unchanged whereas other regions are scaled. In case of non-linear scaling, the scaling factor is highest at the borders.

The seam carving technique proposed by Avidan and Shamir ${ }^{36}$ enables a content-aware adaptation of the resolution of images. The idea is to identify a path (seam) of 8-connected pixels which has a low relevance for the content of an image. Depending on the desired size of the adapted image, several paths are identified and the path pixels are removed in case of a reduction of the image size. An enlargement of an image is also possible by duplicating path pixels.

The following constraints must be regarded to avoid artifacts in the final image. The pixels of a horizontal or vertical seam must be 8-connected (see Fig. 7 for an example of vertical seams). An energy function defines which pixels should be selected for a seam. Avidan and Shamir evaluated the performance of several energy functions and recommended the usage of the gradient. The energy of a seam is defined as the sum of all absolute gradient values of two adjacent path pixels. The seams are sorted in ascending order, so that the first seam contains the lowest overall energy value. If the size of an image is reduced by $N$ pixels, the pixels of the first $N$ seams are removed and all pixels are shifted horizontally or vertically to fill the gaps.

Compared to other approaches, the seam carving technique works particularly well on outdoor pictures and if relevant objects are located at the borders. It works much better than scaling if the aspect ratios of the source and destination images differ significantly. The method also focuses on preserving the important content of the image. On the other hand, images containing straight lines (e. g., houses, a tennis court, text) and well-known objects like faces may become severely distorted.

\section{CHALLENGES FOR THE ADAPTATION}

Algorithms for the adaptation of web pages and images should focus on the characteristic features of a mobile device: screen resolution, CPU power, energy restrictions, and throughput of the wireless network. Additional factors to be considered are user context, user preferences, and the quality of the adapted content.

A minimum resolution of the display is required to visualize complex structures in web pages (e. g., tables, navigation bars, icons), formatting (e. g., headlines or captions), and images. The adaptation of the image resolution is not trivial 
due to the fact that parts of the content may no longer be recognizable in heavily scaled images. On the other hand, cropping of borders removes entire parts of an image. Even advanced image adaptation techniques like seam carving may create unaesthetic images because people or objects may become warped.

Due to limited CPU power in many mobile devices and the demand for energy efficiency, complex analysis and adaptation algorithms cannot run on mobile devices. We have developed a server based adaptation which also guarantees a reduction of the total amount of data to be transmitted over the wireless network. This is especially relevant if other applications and services are used at the same time, e.g., an indoor positioning service.

The adapted content should comply with the expectations of the user. Therefore, their context and preferences have to be considered. Techniques how to handle errors induced by the automatic analysis of web pages or images should be considered, too. Mechanisms should be available to reduce the risk of incorrectly adapted content.

\section{ADAPTATION OF WEB PAGES}

The basic idea of our adaptation system is to analyze the layout of a web page and simplify its structure and formatting rules. For example, all titles are replaced by bold tags and the font size is reduced. The small screen of a mobile device is used much better this way. Visualization tags (e. g., background color), background images and tables are removed, because the width of tables is usually too large for the screen resolution of mobile devices. The reduced complexity of the adapted web pages guarantees that they are visualized efficiently even on mobile devices with limited CPU capacity and that the energy consumption is reduced for network communication and page rendering. Fig. 1 visualizes a typical view of a web page on a mobile device. User evaluations indicated that horizontal scrolling is especially disturbing.
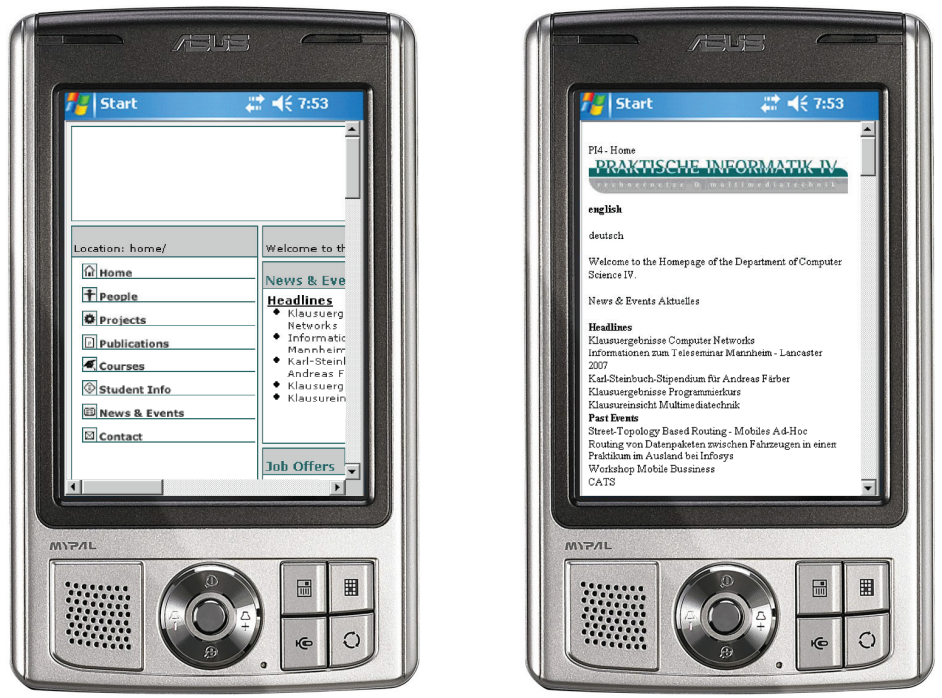

Fig. 1. Typical view of a web page on a mobile device: original view (left), adapted view (right).

The analysis and the adaptation of web pages are processed separately. During the analysis phase, metadata is extracted to analyze the structure of a web page. Typical separators in HTML pages are used to identify sections. Suitable separators are structuring or presentation tags like headings or horizontal lines. We define a minimum and maximum size for each segment. The amount of scrolling should be acceptable if one segment is presented on a mobile device. Short segments are merged with the previous or following segment.

In a second step, a title and a short description is derived for each section. The additional information is required to enable different views of a web page. If an anchor tag is available at the beginning of a section its text is used as title. Otherwise, headings or emphasized text (e.g., text in bold or italic characters) are used. If no title is detected, the first words of a section are selected instead. 
User studies have indicated that the individual preferences of users may vary significantly. Therefore, we support four different views of a web page which are selected based on default user preferences (see Fig. 2). They are: A full view (complete text and relevant images), two navigation views, and an image visualization view. The first navigation view displays titles of sections of a web page which link to the corresponding text. The second navigation view additionally presents a short description. The last view visualizes the resolution adapted images in a web page exclusively.

The full view is generated first. To enable a compact presentation, headings are replaced by bold tags and the size of the characters is set to the default size of the text. Presentation elements like text color, background color and background images are filtered to guarantee a fast visualization on the mobile device. Tables are converted to text because the width of tables is too large in most cases. Images on a web page are adapted (see Section 5), and very small images (icons) are removed. We assume that the semantic content of very small images on web pages is very low. In the last step, additional anchor tags are inserted into the full view to enable direct links to the other views.

Additionally, we have developed a software tool to edit and revise all automatically extracted data. This tool also simulates different mobile devices, so that the user gets a feeling of how an adapted web page will look like. Our semiautomatic approach enables an efficient validation of the automatic analysis step. A user can edit the automatically extracted metadata like title or short description for each section. Sections can be merged or the length of a section can be changed.

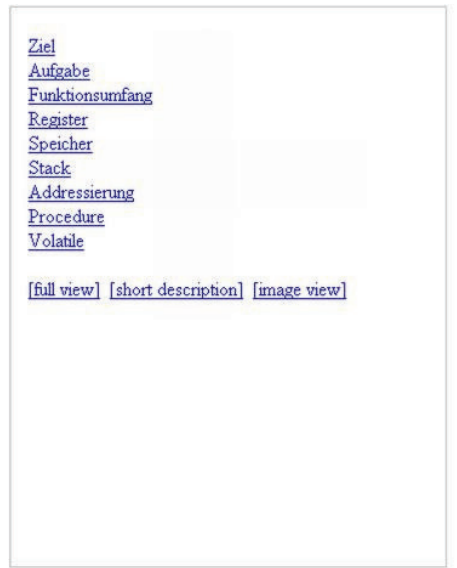

(a)

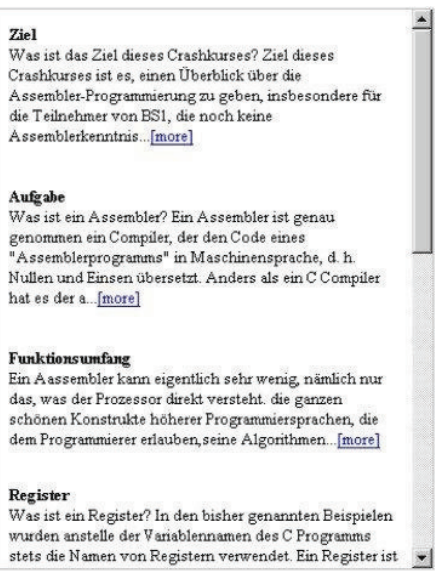

(b)

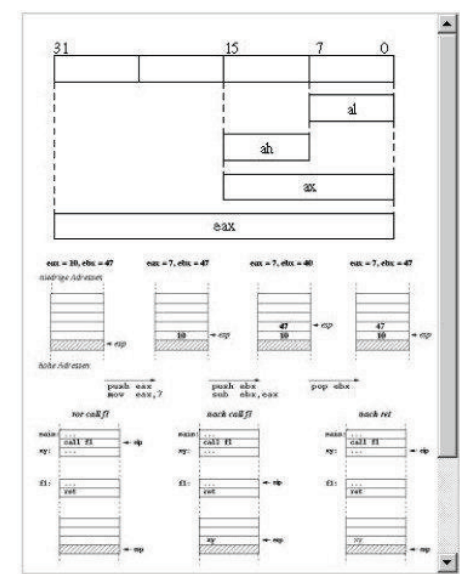

(c)

Fig. 2. Different views of adapted web pages: (a) title view, (b) short description view, (c) image view.

\section{ADAPTATION OF IMAGES}

We have developed a server-based application for the adaptation of images. A client-based adaptation is not feasible because the transfer of large images would cause a lot of traffic in wireless networks, and the analysis and adaptation of an image requires a high amount of computational power. If possible, the analysis of images is done offline, and the results are stored in XML format. The adaptation is done in real-time. To enable a good adaptation, the most relevant region has to be identified in an image. We apply several techniques to identify and validate it:

- Regions without expressive content should not be visualized in the adapted image. Large near-monochrome regions (e.g., sky) adjoining an image border are typical candidates for irrelevant regions.

- Regions with relevant semantic content should be clearly visible in the adapted image. A region should not be part of the adapted image if the semantic content is no longer recognizable due to its small size (e.g., text is no longer readable). We apply algorithms to automatically identify regions with a high contrast, text regions and faces. The adaptation algorithm uses the information about all detected regions. 
- We use a semi-automatic approach to correct obvious errors in the adapted images. Most errors are induced by the automatic analysis. Therefore, we have built a software tool to validate the automatically detected attention objects.

- Inner parts of an image may be removed if relevant objects are located at different borders of an image. We apply the seam carving technique and improve it by using the information about the automatically identified attention objects.

- During the adaptation, the selected region of interest is scaled to the screen resolution of the mobile device. The aspect ratio of the selected region should be similar to the aspect ratio of the adapted image.

In the following subsection, we will describe the image analysis and adaptation techniques in detail.

\subsection{Cropping of irrelevant borders}

Our image adaptation service uses several techniques to select the most relevant content in images. We assume that a mobile device requests an image and specifies the expected resolution. In a first step, border regions are identified, which do not contain relevant semantic content. A typical example is blue sky in the upper region of an image. We crop the borders to remove these regions.

An image is smoothed in a first step with a Gaussian kernel $\sigma=2.0$ to remove noise. Border regions are identified by applying a region growing algorithm on the smoothed image. The maximum difference (sum of absolute differences of RGB values) of the pixel values in a region is defined by $T=30$. Iteratively, we process each border of the image. We analyze one column or row, and count the number of pixels located in each line. If the maximum percentage $P$ of pixels in one line exceed the threshold $T_{P}=0.7$, the current column or row is marked for removal. Relevant parts of an object should not be removed in this step. Therefore, we do not remove all marked rows or columns but search for a local maximum of $P$.

The computational effort to identify irrelevant border regions is very low. Actually, this step reduces the overall time to analyze an image, because advanced image analysis techniques like our face detection algorithm require much more computational effort. Fig. 3 depicts an example which visualizes the identification of irrelevant border regions.

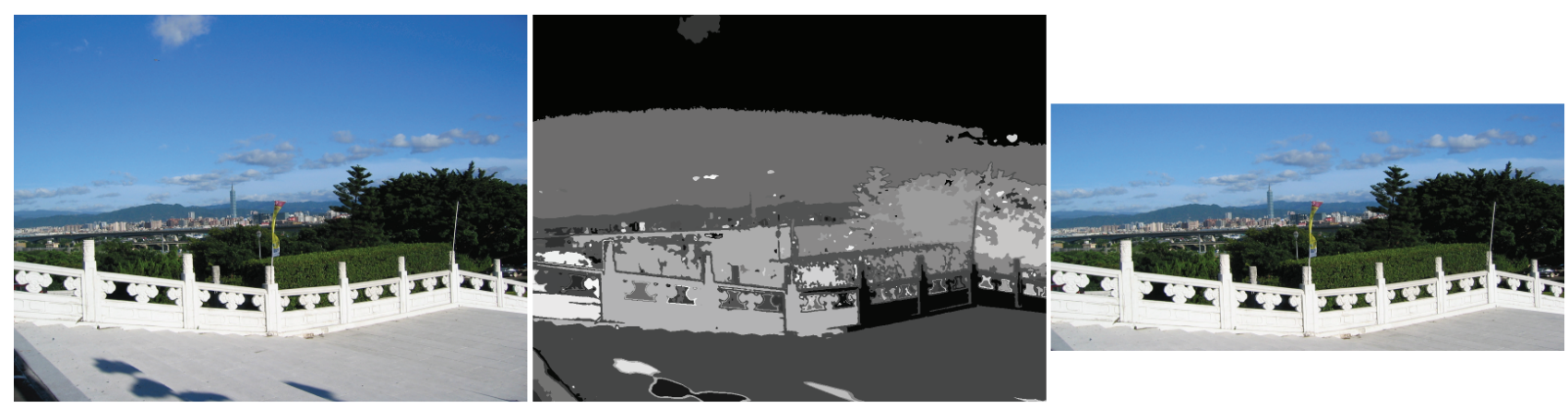

Fig. 3. Automatic cropping of irrelevant borders: original image (left), detected regions (middle), cropped image (right).

\subsection{Selecting a region of interest}

Attention objects are identified in a second step if the resolution of the image is still too high. We use face detection, text detection and contrast based saliency maps to identify important regions in images.

Faces and persons are very important in images and adaptation algorithms should preserve them if possible. We use the face detector published by Rowley et al. ${ }^{37}$ which is based on a neural network. We have implemented the face detector and trained our own network with more than 7,500 faces.

Our text detection system ${ }^{38}$ identifies candidates for text regions first. We assume that each text line contains at least several characters. To locate a text region in an image we use the technique presented by Sato et al.: ${ }^{39}$ Regions with high contrast and sharp edges are detected. A 3x3 horizontal filter with binary thresholding is applied to the image, and 
connected text blocks are identified. If this region suffices certain constrains, the bounding box of this region is classified as text region. In images with complex backgrounds the bounding box may include more than one text line. The next step locates the upper and lower border of each text line by analyzing horizontal projection profiles. The profiles are generated by summarizing the absolute horizontal derivatives. High values indicate a text line, low values background regions. The top and bottom of a text line can be identified by analyzing the local peaks of the projection profile.
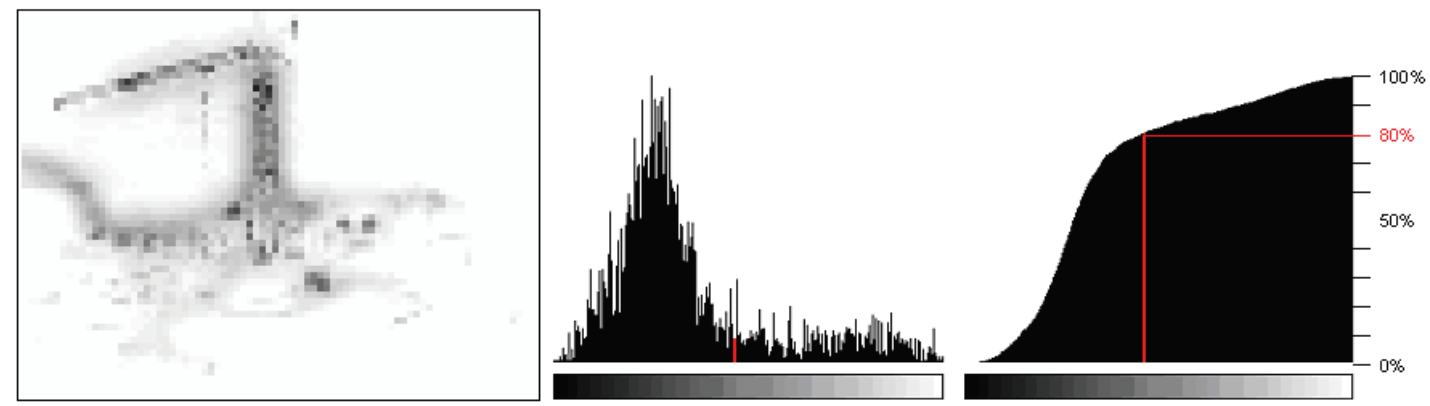

Fig. 4. Detection of regions with high contrast: saliency map (left), histogram (middle), cumulated histogram and derived threshold (right).

Regions with a high contrast are usually much more relevant than homogeneous regions. We identify these regions by analyzing saliency maps. In a first step, we scale the image to a predefined size by averaging the pixel values in a block. We reduce noise and the computational effort this way. We use a method that is similar to the intensity maps introduced by Itti et al.. ${ }^{31}$ The idea is to identify locations in an image which locally stand out from their surrounding. Several scaled versions of the image are generated by using Dyadic Gaussian pyramids. The images are rescaled by linear interpolation and difference images are calculated. The differences are merged into one contrast-based saliency map which describes local discontinuities. Fig. 4 (left) depicts an example of a saliency map. The histogram of the saliency map is used to derive the most relevant region of interest. Therefore, we analyze its cumulated histogram (Fig. 4, right) and select all pixels above the empirically defined threshold $T_{S}=0.8$. The example in Fig. 5 visualizes the automatic detection and adaptation of an image based on saliency maps.

Based on the automatically detected attention objects (faces, text regions and regions based on saliency maps) one region of interest (ROI) is selected for presentation. Each attention object is characterized as a rectangle. We assume a proportional coherence between the size of an attention object in the adapted image and the visual information in the image. A minimal perceptible size is defined for each feature. The content is no longer recognizable if the size of a feature drops below this value. Additionally, we can define an upper size for each feature (maximal reasonable size). For instance, if the font size of a text is readable, an additional enlargement of the characters does not provide new information.

It is the goal to identify the position and size of the region $R$ which maximizes the visible information in the adapted image. The information $V_{\text {sum }}(R)$ is defined as the sum of the attention objects in the selected region $R$ :

$$
\begin{gathered}
V_{\text {sum }}(R)=\sum_{i} S_{i}(R) \cdot V_{i}(R) \quad \text { with } \\
V_{i}(R)= \begin{cases}\frac{H_{\max }}{H_{i}(R)} & H_{i}(R)>H_{\max }, \\
\frac{H_{\text {max }}(R)}{0} & H_{\min } \leq H_{i} \leq H_{\max },\end{cases} \\
S_{i}(R)= \begin{cases}1 & \text { if } V_{i} \text { is part of } R, \\
0 & \text { else. }\end{cases}
\end{gathered}
$$



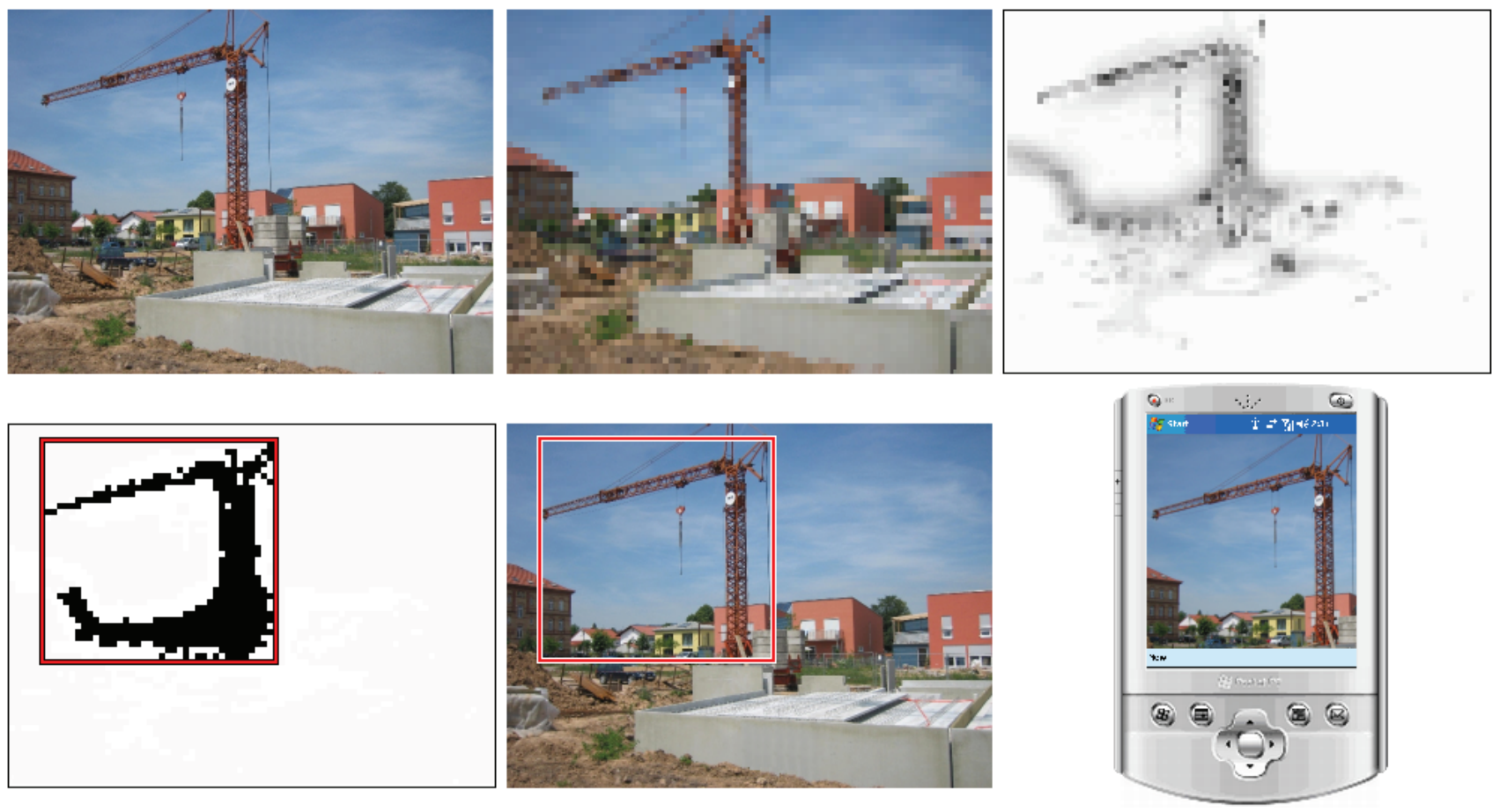

Fig. 5. Automatic detection of relevant regions based on saliency maps.

$V_{i}(R)$ estimates the information of the attention object $i$ for region $R$. An object is used only if it is completely included in the selected region. The binary variable $S_{i}(R)$ specifies whether the region of the attention object is totally included in the region or not. The thresholds $H_{\min }$ and $H_{\max }$ define the minimal perceptible size and maximal reasonable size of attention objects. Both values depend on the characteristics of the display and individual user preferences.

The calculation of all positions and sizes for region $R$ is not possible due to the large number of combinations. We assume that each border of the selected region must match with at least one border of an attention object. This is true, because if we reduce the size of the region marginally, at least one object $i$ will no longer be included in the region and $V_{\text {sum }}(R)$ drops approximately by $V_{i}(R)$. A very small increase of the size of the region leads to a higher scaling factor, and the values of all attention objects in this region drop. $V_{\text {sum }}(R)$ is not necessarily optimal (e.g., in case of text regions) because the maximal reasonable size $H_{\max }$ reduces the visible information of a region. After the initialization of $R$, the size of the region is enlarged until $V_{\text {sum }}(R)$ reaches a local maximum.

\subsection{Semi-automatic refinement}

We assume that we cannot avoid errors during the automatic analysis. Therefore, we propose a semi-automatic approach. We have specified XML schemas based on MPEG-7 and MPEG-21 to store the information about the attention objects in an image. We have developed a software tool, which enables a user to validate, add, delete, or modify the automatically detected objects in a convenient way. Additional information like the maximum acceptable scaling factor may be added for each object.

Fig. 6 visualizes how a user can modify the automatically detected attention objects in images. General information like filename, title, image resolution, and image orientation is stored for each image. Additionally, an arbitrary number of regions of interest can be defined. Each region is characterized by a textual description, a value that characterizes its relevance, and a maximum scaling factor. The metadata is stored in XML format and used for the adaptation. 


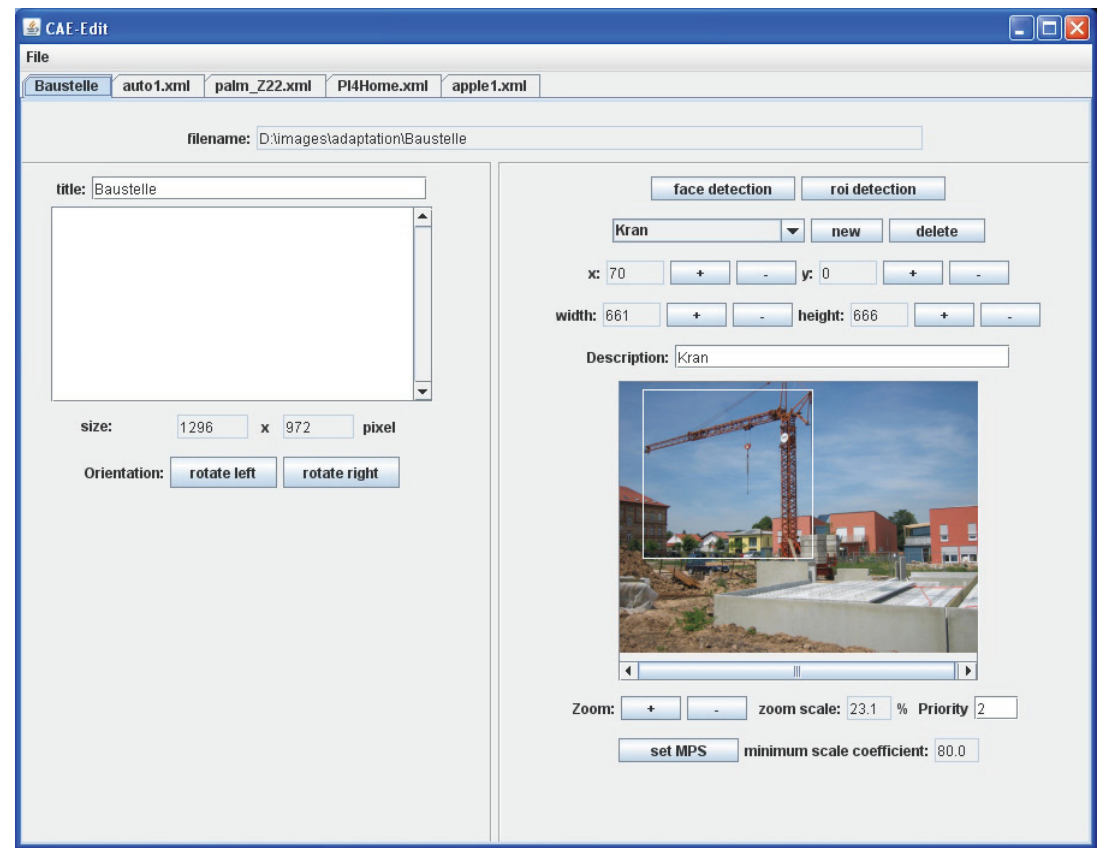

Fig. 6. A software tool enables the semi-automatic refinement of metadata.

\subsection{Removing inner parts of an image}

It is often not possible to select a suitable region of interest if two or more attention objects are located at different borders of an image. Inner parts of an image may be removed by using the seam carving technique. ${ }^{36}$ The idea is to identify a horizontal or vertical path of 8-connected pixels and remove it. By selecting the path with the lowest energy value relevant semantic objects are usually preserved. Fig. 7 depicts an example of the seam carving technique in which vertical seams are removed.

We use the automatically detected attention objects to modify the seam carving algorithm. The idea is to increase the energy values of attention objects. The seams will avoid these regions and preserve the semantic content in the image.
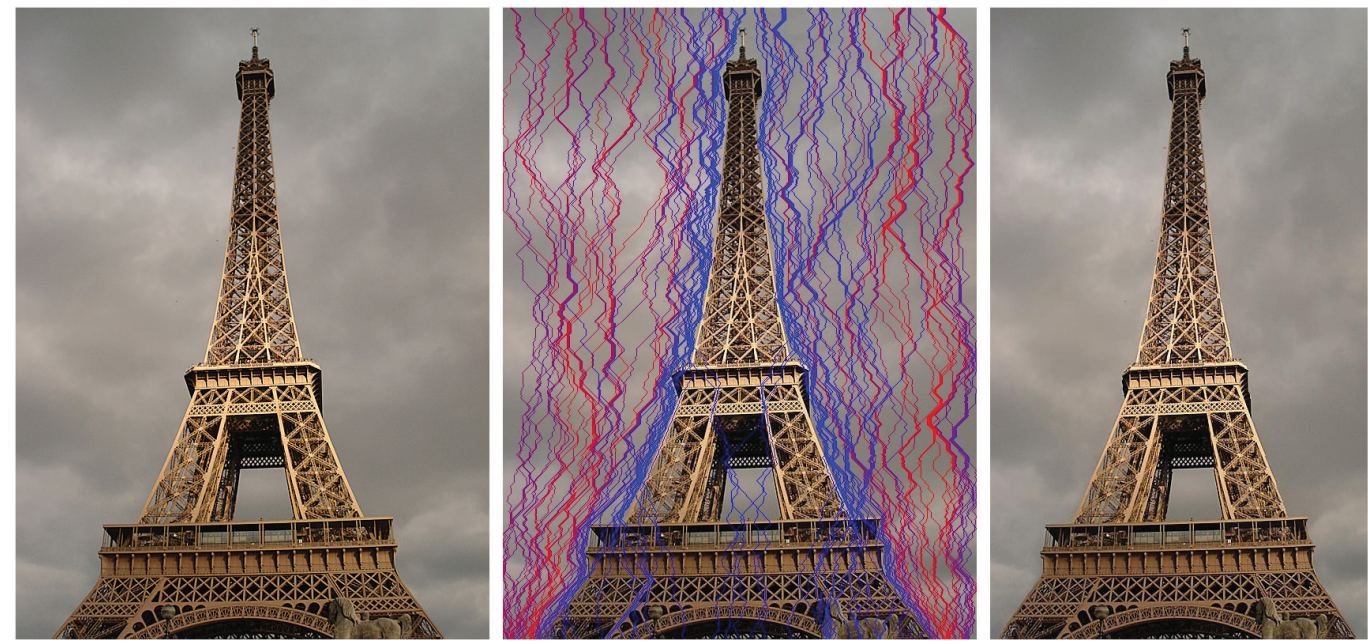

Fig. 7. Removing inner parts of an image based on the seam carving technique: original image (left), detected seams (middle), adapted image (right). Red seams (low energy values) are removed first. 


\subsection{Automatic image adaptation}

The adaptation of the content for different mobile devices is fully automatic. The different techniques are applied one by one until the image resolution fits. Irrelevant borders are identified and cropped first. If the resolution is still too large, a region of interest based on attention objects is selected. Inner parts of an image may be removed if the image is significantly larger than the expected resolution or if the aspect ratios differ significantly. Linear interpolation is used to scale the image to its final resolution.

\section{EVALUATION}

We have carried out three user studies to evaluate the quality of the adaptation system. Questions regarding software ergonomics, quality of the adapted content, and perceived benefit of the adaptation were asked. The feedback about the implemented adaptation technique for web pages and images is very positive. Especially the combination of automatic and semi-automatic algorithms and the easy way to correct the automatically generated metadata is a great benefit of this system.

We have simulated 20 different mobile devices with resolutions between 160x160 and 640x480 pixels. In a first test, ten users evaluated the quality of the automatic adaptation algorithm without semi-automatic refinement. Each person examined images which had been adapted for randomly selected devices. The users had to grade the quality based on a scale between 1.0 (very good) and 6.0 (insufficient). The average value for the automatic adaptation of images is 2.4. Two users classified the results barely adequate. The reason for this low rating was the fact that the quality of some adapted images was very poor especially if important objects were missing or if parts of them had been cropped.

Each test user had to correct or add the automatically detected attention objects in a second evaluation. The adapted images were presented again, and the quality was considered much higher this time (average value of 1.9). Some users complained that a minimum border should be added to each attention object especially if a face is located at the border of an image. The image quality is 'acceptable' for two users; the other users grade it much better (between good and very good).

We tested the quality of the adaptation algorithm for web pages in a separate evaluation. Ten users participated in this evaluation. The quality of the adapted web pages was rated much higher (average value of 1.5). Several users recommended that the user interface of the software tool to modify the sections of an HTML page should be simplified. We explicitly asked the users about the different views. Half of the users liked them very much, but three users did not switch to another view because they were familiar with one view from their Web browser and did not need additional information to browse a web page.

All users confirmed that they see the great benefit such an adaptation system offers. Some users remarked that the manual effort should be as low as possible.

\section{CONCLUSIONS AND OUTLOOK}

In this paper, we have presented our new visualization service for web pages and images. We simplified the layout and structure of a web page to achieve a compact presentation. Our image adaptation service combines several techniques. In a first step, irrelevant borders are identified and cropped. If the resolution is still too large, a region of interest based on attention objects is selected. Inner parts of an image may be removed by seam carving if the image is much larger than the expected resolution or if the aspect ratios differ significantly. Linear interpolation is used in a last step to scale the image to its final resolution. Additionally, we have developed a software tool to validate, add, delete, or modify all automatically extracted data. This tool also simulates different mobile devices, so that the user gets a feeling of how an adapted web page or image will look like. We have performed user studies to evaluate the quality of our adaptation system. The users confirmed that they see a great benefit such an adaptation system offers.

Our long-term goal is to provide generic tools for the automatic adaptation of multimedia content. We will focus on the adaptation of audio and video streams in the near future. 


\section{ACKNOWLEDGEMENTS}

We would like to thank Victor Schan for his help. He developed the software tool to edit and revise all automatically extracted data. Furthermore, the authors acknowledge the financial support granted by the Deutsche Forschungsgemeinschaft (DFG).

\section{REFERENCES}

[1] Kopf, S., King, T., Bostan, P., Lemelson, H., Deibert, S., and Effelsberg, W., "Fundamental Services for ContextSensitive Mobile Applications," in International Workshop on Human Control of Ubiquitous Systems (HUCUBIS), Dublin, Ireland, pp. 1-6 (2008).

[2] Mohan, R., Smith, J., and Li, C. S., "Adapting Multimedia Internet Content For Universal Access," IEEE Transactions on Multimedia 1(1), pp. 104-114 (1999).

[3] Noble, B. D., Satyanarayanan, M., Narayanan, D., Tilton, J. E., Flinn, J., and Walker, K. R., "Agile ApplicationAware Adaptation for Mobility," in 16th Symp. on Operating System Principles (SOSP), Saint-Malo, France (1997).

[4] Han, R., Bhagwat, P., LaMaire, R., Mummert, T., Perret, V., and Rubas, J., "Dynamic Adaptation in an Image Transcoding Proxy for Mobile WWW Browsing," IEEE Personal Communication 5(6), 8-17 (1998).

[5] Lum, W. Y., and Lau, F.C.M., "A Context-Aware Decision Engine for Content Adaptation," IEEE Pervasive Computing 1(3), 41-49 (2002).

[6] Lei, Z., and Georganas, N. D., "Context-based media adaptation in pervasive computing," in Proceedings of IEEE Canadian Conference on Electrical and Computer (CCECE'01), Toronto (2001).

[7] Cardellini, V., Yu, P. S., and Huang, Y. W., "Collaborative proxy system for distributed Web content transcoding," in Proceedings of 9th International ACM Conference on Information and Knowledge Management, Washington, DC, pp. 520-527 (2000).

[8] Kopf, S., and Effelsberg, W., "Mobile cinema: canonical processes for video adaptation," Multimedia Systems 14(6), pp. 369-375 (2008).

[9] Kopf, S., Haenselmann, T., Farin, D., and Effelsberg W., "Automatic Generation of Summaries for the Web," in Proceedings of SPIE, Storage and Retrieval for Media Databases 5307, San José, CA, pp. 417-428 (2004).

[10] Kopf, S., Lampi, F., King, T., and Effelsberg W., "Automatic Scaling and Cropping of Videos for Devices with Limited Screen Resolution," in Proceedings of ACM Multimedia, Santa Barbara, CA, USA (2006).

[11] Rowe, N., "Critical Issues in Content Repurposing for Small Devices," in Encyclopedia of Multimedia Technology and Networking, ed. M. Pagani, Hershey, PA: The Idea Group (2005).

${ }^{[12]}$ Fox, A., Gribble, S. D., Chawathe, Y., and Brewer, E. A., "Adapting to Network and Client Variation Using Infrastructural Proxies: Lessons and Perspectives," IEEE Personal Communication 5(4), pp10-19 (1998).

${ }^{[13]}$ Kopf, S., and Effelsberg W., "Color Adaptation of Videos for Mobile Devices," in Proceedings of IS\&T/SPIE conference on Multimedia on Mobile Devices 6507, San Jose, CA, USA, pp. 1-12 (2007).

[14] Kopf, S., King, T., Lampi, F., and Effelsberg, W., "Video Color Adaptation for Mobile Devices," in Proceedings of ACM Multimedia, Santa Barbara, CA, USA (2006).

[15] Chen, J. L., Zhou, B. Y., Shi, J., Zhang, H.-J., and Wu, Q. F., "Function-based Object Model Towards Website Adaptation," in Proceedings of $W W W$, Hong Kong (2001).

[16] Gu, X. D., Chen, J. L., Ma, W. Y., and Chen, G. L., "Visual Based Content Understanding towards Web Adaptation," in 2nd Intl. Conf. on Adaptive Hypermedia and Adaptive Web Based Systems, Malaga, Spain, pp. 164173 (2002).

[17] Rahman, A. F. R., Alam, H., Hartono, R., and Ariyoshi, K, "Automatic Summarization of Web Content to Smaller Display Devices,", in Post Presentations of 6th International Conference on Document Analysis and Recognition, Seattle, The United States (2001).

[18] Bickmore, T., Girgensohn, A., and J. Sullivan, "Web page filtering and Re-authoring for Mobile Users," The Computer Journal 42(6), pp. 334 - 346 (1999).

[19] Buyukkokten, O., Garcia-Molina, H., and Paepcke, A., "Seeing the Whole in Parts: Text Summarization for Web Browsing on Handheld Devices," in Proceedings Of the World Wide Web Conference, ACM Press (2001).

[20] Penn, G., Hu, J., Luo, H., and Mcdonald, R., "Flexible Web Document Analysis for Delivery to Narrow-Bandwidth Devices," in Proceedings International Conference on Document Analysis and Recognition, IEEE CS Press, pp. $1074-1078$ (2001). 
[21] Bickmore, T. W., "Device-independent access to the World Wide Web," in Proceedings of WWW Conference, pp. $655-663$ (1997).

[22] Perkowitz, M., “Towards adaptive web sites: Conceptual framework and case study,” AI Journal 118(1,2) (2000).

[23] Huang, A. W., and Sundaresan, N., "Aurora: A Conceptual Model for Web-Content Adaption to Support the Universal Usability of Web-based Services," in Proceedings on the 2000 ACM conference on Universal Usability, pp. 124-131 (2000).

[24] Chen, J., Zhou, B., Shi, J., and Zhang, H., Fengwu, Q., "Function-Based Object Model Towards Website Adaption," in Proceedings of the 10th international conference on World Wide Web, pp. 587-596 (2001).

[25] Maekawa, T., Arase., Y., Hara, T., Uemukai, T., and Nishio, S., "A Web Browsing System based on Adaptive Presentation of Web Contents for Cellular Phones," in Proceedings of the 2006 International Cross-Disciplinary Workshop on Web Accessibility (W4A), Vol. 134, pp. 86-89 (2006).

[26] Blekas, A., Garofalakis, J., and Stefanis, V., "Use of RSS feeds for Content Adaption in Mobile Web Browsing," in Proceedings of the 2006 International Cross-Disciplinary Workshop on Web Accessibility (W4A), Vol. 134, pp. 7985 (2006).

[27] $\mathrm{Hu}, \mathrm{J}$. , and Bagga, A., “Categorizing Images in Web Documents," IEEE MultiMedia 11(1) (2004).

[28] Kasik, D., "Strategies for Consistent Image Partitioning," IEEE Multimedia 11(1), pp. 32 - 41 (2004).

[29] Rist, T., and Brandmeir, P., "Customizing Graphics for Tiny Displays of Mobile Devices," in Mobile HCI '01 (2001).

[30] Suh, B., Ling, H., Bederson, B., and Jacobs, D., "Automatic thumbnail cropping and its effectiveness," in Proceedings of the 16th annual ACM symposium on User interface software and technology, ACM Press, New York, NY, USA, pp. 95-104 (2003).

[31] Itti, L., Koch, C., and Neibur, E., "A model of saliency-based visual attention for rapid scene analysis," IEEE Transactions on Pattern Analysis and Machine Intelligence 20(11), pp. 1254-1259 (1999).

[32] Santella, A., Agrawala, M., Decarlo, D., Salesin, D., and Cohen, M., "Gaze-based interaction for semi-automatic photo cropping," in ACM Human Factors in Computing Systems (CHI), pp. 771-780 (2006).

[33] Setlur, V., Takagi, S., Raskar, R., Gleicher, M., and Gooch, B., "Automatic image retargeting," in Proceedings of International Conference on Mobile and Ubiquitous Multimedia, pp. 247-250 (2005).

[34] Liu, H., Xie, X., Ma, W., and Zhang, H., "Automatic browsing of large pictures on mobile devices," in 11th ACM International Conference on Multimedia (2003).

[35] Liu, F., and Gleicher, M., "Automatic image retargeting with fisheye-view warping," in ACM UIST, pp. 153-162 (2003).

[36] Avidan, S., and Shamir, A., "Seam carving for content-aware image resizing," ACM Transactions on Graphics (SIGGRAPH 2007) 26(3) (2007).

[37] Rowley, H., Baluja, S., and Kanade, T., "Neural network-based face detection," IEEE Transactions on Pattern Analysis and Machine Intelligence 20, pp. 23-38 (1998).

[38] Kopf, S., Haenselmann, T., and Effelsberg, W., "Enhancing Curvature Scale Space Features for Robust Shape Classification", in Proceedings of IEEE International Conference on Multimedia and Expo (ICME, Amsterdam, Netherlands), pp. 1-4 (2005).

[39] Sato, T., Kanade, T., Hughes, E., Smith, M., and Satoh S., "Video OCR: Indexing digital news libraries by recognition of superimposed captions," ACM/Springer Multimedia Systems 7, pp. 385-395. ACM Press (1999). 